\title{
The role of heat shock proteins in antigen cross presentation
}

\author{
Ayesha Murshid ${ }^{1}$, Jianlin Gong ${ }^{2}$ and Stuart K. Calderwood ${ }^{1}$ * \\ ${ }^{1}$ Molecular and Cellular Radiation Oncology, Beth Israel Deaconess Medical Center, Harvard Medical School, Boston, MA, USA \\ ${ }^{2}$ Boston University Medical Center, Boston, MA, USA
}

\author{
Edited by: \\ Christian Kurts, Friedrich \\ Wilhelms-Universität Bonn, Germany \\ Reviewed by: \\ Emmanuel Wiertz, University Medical \\ Center Utrecht, Netherlands \\ Willem van Eden, Utrecht University, \\ Netherlands \\ Jonathan Yewdell, National Institute of \\ Allergy and Infectious Diseases, USA \\ ${ }^{*}$ Correspondence: \\ Stuart K. Calderwood, Beth Israel \\ Deaconess Medical Center, Harvard \\ Medical School, 99 Brookline Avenue, \\ Boston, MA 02215, USA \\ e-mail:scalderw@bidmc.harvard.edu
}

Heat shock proteins (HSPs) are molecular chaperones that bind tumor antigens and mediate their uptake into antigen presenting cells. HSP-antigen complexes are then directed toward either the MHC class I pathway through antigen cross presentation or the conventional class II pathway, leading to activation of T cell subsets. Uptake of HSP-chaperoned polypeptides can involve both receptor-mediated and receptor-independent routes, and mechanisms of antigen sorting between the Class I and II pathways after uptake are currently under investigation. The processes involved in internalization of HSP-antigen complexes differ somewhat from the mechanisms previously determined for (unchaperoned) particulate and free soluble antigens. A number of studies show that HSP-facilitated antigen cross presentation requires uptake of the complexes by scavenger receptors (SR) followed by processing in the proteasome, and loading onto MHC class I molecules. In this review we have examined the roles of HSPs and SR in antigen uptake, sorting, processing, cell signaling, and activation of innate and adaptive immunity.

Keywords: heat shock proteins, antigen cross presentation, CTL response, scavenger receptor, antigen presenting cells, soluble vs. particulate antigen, anti-cancer vaccine, tumor immunity

\section{INTRODUCTION}

Heat shock proteins (HSPs) are a class of polypeptides powerfully induced by heat shock that mediate profound levels of stress resistance (Craig, 1985; Ellis, 2007). HSPs are molecular chaperones, binding to (holding) and refolding other cellular polypeptides (clients) with aberrant conformations (Ellis, 2007). There are a number of families of molecular chaperone families $(\mathrm{a}-\mathrm{d})$, with members of class a (Hsp70, Hsp110, GRP170) and class c (Hsp90, Grp94/Gp96) thought to be of particular significance in tumor immunology (Murshid et al., 2011c). (Grp is the abbreviation for glucose regulated protein and such proteins are retained in the endoplasmic reticulum, ER.) The molecular chaperone activity of class a and class $\mathrm{c}$ chaperones appears to be conferred by two functional domains: a dedicated peptide binding domain that seizes client polypeptides and an ATPase domain (Scheibel et al., 1998; Mayer and Bukau, 2005; Vogel et al., 2006). HSPs are allosteric molecules, one domain reciprocally affecting the other, and when polypeptide moieties bind to the peptide binding domain, ATP is hydrolyzed to ADP and orthophosphate and when ATP binds, associated peptides are discarded. These properties have been intensely studied for Hsp70 and Hsp90 and are largely inferred for the sibling proteins. The ability to hold polypeptide clients appears to correlate with the size of class a chaperones, the smaller class a member (Hsp70) binding to smaller peptides with medium affinity while larger class a chaperones (Hsp110 and Grp170), with very large peptide binding domains, bind tightly to their clients, including peptides and whole proteins (Oh et al., 1999; Park et al., 2006).

Heat shock protein-peptide complexes (HSP.PC) can be used as vaccines to elicit antigen-specific cytotoxic lymphocytes (CTL) responses (Srivastava, 2000; Manjili et al., 2002; Wang et al., 2003; Enomoto et al., 2006; Gong et al., 2010). In order for polypeptides bound to HSPs to activate adaptive immunity, associated antigens must be internalized by antigen presenting cells (APC) and inserted into the antigen presentation pathways. Indeed, HSPs have been shown to be taken up by dendritic cells (DC), the most efficient professional APC (Steinman et al., 1983; Heath and Carbone, 2009). Antigen presentation occurs through a number of pathways. Intracellular proteins were shown to be processed by digestion through the multiple protease activities in the proteasome and antigens presented on the cell surface by major histocompatibility class I (MHCI) molecules found in all cells and thus displayed to $\mathrm{CD} 8+\mathrm{T}$ cells to permit immunosurveillance (Neefjes and Momburg, 1993). By contrast, exogenous antigens after internalization into immune cells are processed in lysosomes and presented on the cell surface by major histocompatibility class II (MHCII) molecules restricted to cells of the immune system (Cresswell, 1994). It was subsequently shown that another antigen presentation pathway exists permitting external antigens to enter the MHC class I pathway (Norbury et al., 2002, 2004). This process, antigen cross presentation permits external antigens to be presented by APC in the context of MHC class I and activate CD8+ CTL to kill virus infected or malignant cells (Norbury et al., 2002, 2004; Figure 1). Antigen cross presentation was shown to be a complex process requiring external antigens to enter cells, penetrate sites for protein processing, and associate with MHC class I molecules in intracellular vesicular structures although many aspects of this process are incompletely understood. We aim to elucidate some of the mechanisms by which antigens bound to HSP interact with APC and mediate antigen 


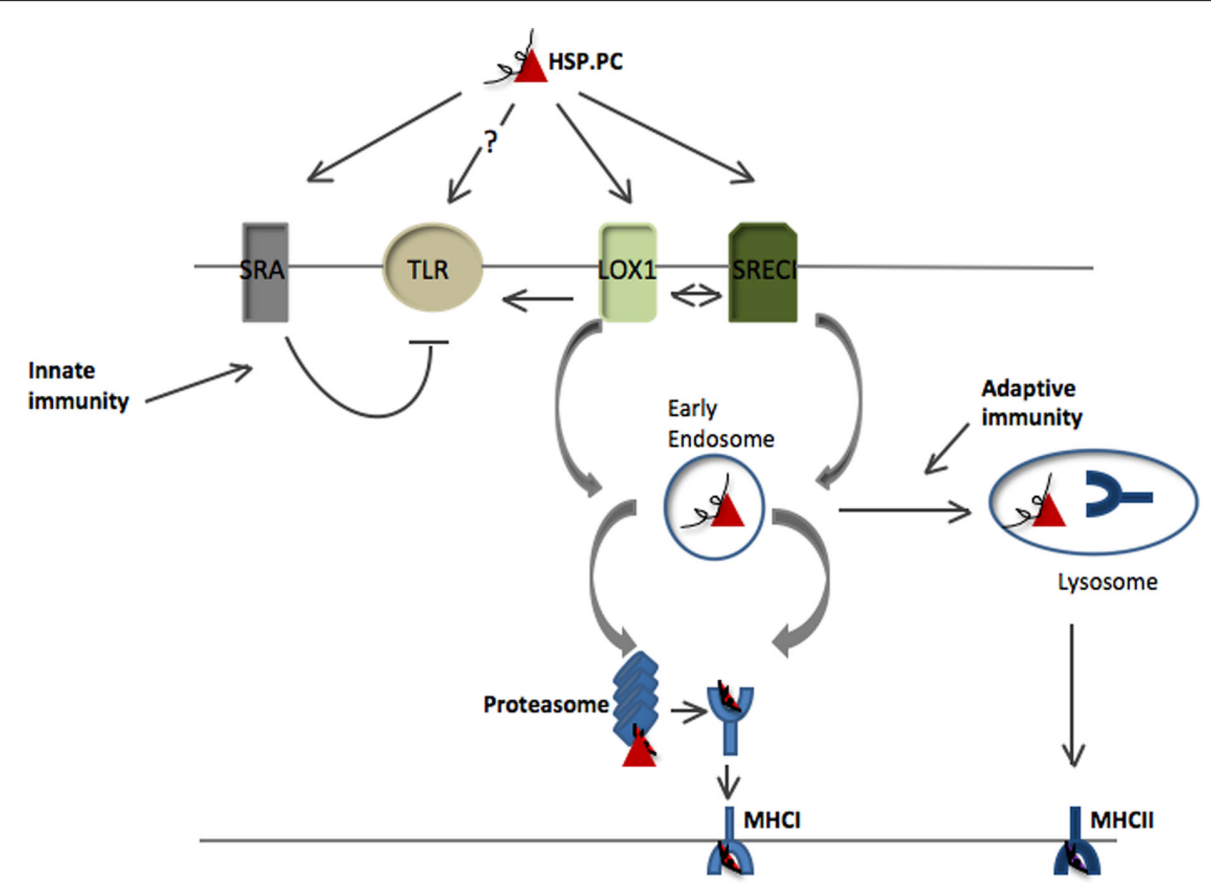

FIGURE 1 | Antigen presentation pathways for HSP-bound polypeptides. HSP.PCs bind to surface receptors on DC including SRECI and LOX-1 and are internalized in complexes with the receptors. Binding to these receptors may also trigger secondary activation of Toll-like receptors (TLR2 or TLR4) that may amplify antigen cross presentation. Alternatively some studies suggest direct binding of HSPs to TLR. HSP binding to cell surface SRA/CD204 is inhibitory to TLR4 activity and likely antagonizes the immune responses induced by HSPs through LOX-1 or SRECI. HSP.PC are internalized by SRECI or LOX-1 into endosomes with the subsequent release of the peptides from the HSP.PC complex. Such peptides are then processed either within endosomes or undergo trafficking to the cytosol where they encounter the proteasomal system. Processed peptides are then loaded either onto $\mathrm{MHC}$ class I in the $\mathrm{ER}$ or phagosomes or onto $\mathrm{MHC}$ class II molecules in lysosomes and presented to, respectively CD8+T cells and CD4+T cells. The triage mechanisms directing HSP-chaperoned peptides toward either of these two pathways are currently not known. cross presentation as compared with the pathways utilized for unchaperoned antigens.

\section{HSPS IN TUMOR IMMUNITY AND ANTI-CANCER VACCINES: COMMON AND UNCOMMON ANTIGENS}

When HSPs are extracted from cancer cells under controlled conditions and introduced into tumor bearing hosts they can induce immunity to the parent tumor cells (Srivastava, 2002; Manjili et al., 2003; Wang et al., 2003, 2010; Murshid et al., 2011c). The major paradigm for using tumor derived HSP as vaccines involves their assumed ability to bind to antigenic oligopeptides (Srivastava, 2002; Murshid et al., 2011c). This hypothesis takes into account the chaperone properties of the HSPs mentioned above and their relative abundance inside cells and is supported by a considerable body of data. However some controversy remains, particularly for the ER resident class c chaperone Grp94 (Gp96; Chandawarkar et al., 1999, 2004; Jockheck-Clark et al., 2010). Although some indirect studies suggested that Grp94 could bind to antigenic peptides in vitro, these findings have been disputed (Ying and Flatmark, 2006). In addition, this protein has been shown to initiate tumor rejection in the absence of bound peptides, presumably through stimulation of innate immunity (Nicchitta, 2003). However these drawbacks may not apply to the other class $a$ and $c$ chaperones. Hsp70 has for instance been shown to bind to oligopeptides in vitro by phage display and affinity chromatography approaches and in vivo by mass spectrometry (Flynn et al., 1989; Fourie et al., 1994; Gragerov and Gottesman, 1994; Grossmann et al., 2004).

Heat shock protein levels are elevated in many tumor types due to the increased requirement for molecular chaperones needed to stabilize the abundant mutant and over-expressed oncoproteins found in cancer (Calderwood et al., 2006; Calderwood and Gong, 2011). The expansion of molecular chaperone clients during stress was shown previously to trigger expression of HSPs due to elevated transcription and selective translation (Calderwood et al., 2010). HSP-oncoprotein complexes thus accumulate in abundant levels in such tumor cells in response to the increased folding burden and can be used to prepare chaperone-based vaccines: this is the major rationale behind using HSPs in tumor immunotherapy (Srivastava and Old, 1988; Kamal et al., 2003; Murshid et al., 2011c). The multiple mutations found in cancer genomes appear to be required to overcome the matrix of regulatory barriers to transformation and progression that characterize normal cells (Vogelstein and Kinzler, 1993; Hanahan and Weinberg, 2000). Alterations such as mutation and overexpression occur in growth factor receptors and downstream signaling pathways and permit cytokine-independent growth in cancer. In addition, mutations in tumor suppressor genes override their inhibitory effects of their gene products on cancer (Hanahan and Weinberg, 2000). However not all mutations are equal. Many cells in the tumor population may be unable to initiate efficient tumor formation 
and tumorigenesis appears to be maintained by driver mutations in minority clones of tumor-initiating cells (TIC; Carter et al., 2009; Hanahan and Weinberg, 2011). Thus many of the random mutations accumulating in the large non-TIC population may have passenger status and are unlikely to select for malignancy. A high proportion of the antigenic epitopes in individual cancers could therefore be unique and not shared with other tumors. However, many tumors express common antigens (such as HER2-neu and MUC1 in breast cancer) or re-express embryonic and developmental genes such as Mart1 and gp100 common to many cancers (Engelhard et al., 2002). For tumor immunotherapy in patients, Grp94 and Hsp70 vaccines have been used in an autologous mode, with the patients receiving HSP complexes derived from their own primary tumors for immunotherapy (Srivastava and Old, 1988). This approach was based on experimental studies of chemically induced rodent cancers, in which Grp74 and Hsp70 vaccines were shown to be effective only in an autologous context and were not cross reactive even with tumors from matched animals bearing tumors induced by the same carcinogen (Udono and Srivastava, 1993; Chandawarkar et al., 2004).

Characterizing the antigenic peptide repertoires associated with individual HSPs extracted from tumor cells has however rarely been attempted. Thus although it is suspected that the individual class a and class $\mathrm{c}$ HSPs chaperone a wide spectrum of potential antigenic epitopes that may be representative of the cell proteome and that individual HSPs may favor distinct subpopulations of such peptides, these issues have not been comprehensively studied and the question remains open.

\section{PATHWAYS OF ANTIGEN CROSS PRESENTATION: SOLUBLE, PARTICULATE, AND HSP-CHAPERONED ANTIGENS}

Antigen presenting cells utilize at least two pathways for sampling external antigens and displaying them to T lymphocytes. These are the MHC class I and MHC class II pathways. However, the mechanisms used for sorting and trafficking of antigens between the MHC class I or class II pathways varies according to the physical nature of the antigen (Inaba and Inaba, 2005). Particulate and soluble antigens are dealt with quite differently.

(a) Particulate antigens are taken up by phagocytosis and enter vesicular structures known as phagosomes (Ackerman et al., 2003; Cresswell et al., 2005; Inaba and Inaba, 2005). Triage of antigens between the Class I and Class II pathways then appears to involve close regulation of the intraphagosomal $\mathrm{pH}$. The previously acidic $\mathrm{pH}$ of the phagosome is initially increased, favoring antigen processing through the Class I pathway. After some minutes, these phagosomes are reacidified, permitting antigen processing toward the Class II pathway (Inaba and Inaba, 2005).

Following triage between MHC class I and class II pathways, the cross presentation of particulate antigens involves in itself at least two variant mechanisms. In the Cytosolic Pathway of Antigen Cross Presentation, antigens are taken up into phagosomes and, after partial processing in these organelles, exported into the cytoplasm where they are hydrolyzed into short peptides within the proteasome. The processed peptides are then re-imported into the ER by transporter associated with antigen presentation (TAP), enter the conventional antigen presentation pathway, and become loaded onto appropriate MHC class I molecules (Rock and Shen, 2005). A variation on this mechanism has been proposed in which the antigen processing machinery of the ER becomes associated with phagosomes and loading onto MHC class I occurs in these organelles (Rock et al., 2005).

A second, alternative pathway for cross presentation of particulate antigens has been described, The Vacuolar Pathway, also known as the TAP-independent pathway of cross presentation (Rock and Shen, 2005; Ackerman et al., 2006). This route of antigen presentation is rapid compared to the cytosolic/proteasome pathway and obviates the need for polypeptides to be pumped in and out of the cytoplasm. In this case, antigens are taken up into endosomes and directly processed by endogenous proteases such as the cysteine protease cathepsin S, loaded onto MHC class I molecules recycling from the cell surface and then trafficked back to the surface in recycling endosomes.

(b) Soluble antigens appear to be taken up by APC and partitioned toward the MHC class I and MHC class II pathways by different mechanisms compared to particulate antigens. Sorting to the class I and class II routes occurs at the cell surface through either non-specific macropinocytosis or endocytosis, involving surface receptors. Uptake of antigens directed to the antigen cross presentation pathways may involve $\mathrm{Fc}$ receptors, and c-type lectins including CLEC9A, DC-SIGN, DEC205, and the mannose receptor 1 (Bonifaz et al., 2002; Burgdorf et al., 2006, 2010; Idoyaga et al., 2011; Tacken et al., 2011). C-type lectins have in common the possession of a recognition domain that determines their carbohydrate specificity and have been studied mostly in regard to interaction with pathogens (Geijtenbeek and Gringhuis, 2009). Cross presentation of internalized antigens next involves the two variant pathways described above for particulate antigens after antigen-receptor complexes are internalized into endosomes.

\section{EXTRACELLULAR HSP_PEPTIDE COMPLEXES AND ANTIGEN CROSS PRESENTATION}

The uptake of HSP.PC may involve receptors expressed on the surface of DC and can precede antigen presentation through the Class I and Class II pathways (Jeannin et al., 2005; Theriault et al., 2006; Kurotaki et al., 2007; Murshid et al., 2011b). The receptors involved in this process appear to differ from those that mediate uptake of soluble, unchaperoned antigens, and the c-type lectins involved in the latter process do not seem to be major endocytic receptors for HSP.PC in APC (Theriault et al., 2006). Instead HSPs bind mainly to scavenger receptors (SR) such as SRECI/Scarf1, FEEL-I/Stabilin-1, LOX-1, SRA/CD204, and LRP1/CD91 (Binder et al., 2000; Delneste et al., 2002; Theriault et al., 2006; Murshid et al., 2011b; Figure 1). SR were first characterized in studies of uptake of chemically modified low-density lipoproteins (LDL) by endothelial cells and appear to bind a wide variety of other molecules, including HSPs (Murshid et al., 2011b). However, Hsp70 can be bound by some molecules with c-type lectin specificity, including the natural killer (NK) cell receptor NKG2D; LOX-1 belongs to both SR and c-type lectin families (Theriault et al., 2006; Murshid 
et al., 2011a). As FEEL-1 is not abundantly expressed in DC we have not further pursued its potential role in HSP-mediated antigen cross presentation (Chu, B., Murshid, A., and Calderwood, S. K., unpublished). The status of LRP1/CD91 in HSP binding and cross presentation is somewhat controversial. This protein has been proposed as a common HSP receptor (Binder et al., 2000; Srivastava, 2002). However, LRP1/CD91 is not abundantly expressed in most DC subtypes and binding of Grp94, calreticulin, and Hsp70 to the receptor was not detected in a number of studies (Delneste et al., 2002; Berwin et al., 2003; Walters and Berwin, 2005; Theriault et al., 2006). SRA/CD204 plays a significant immune suppressive function after binding HSPs and other ligands and there is currently no evidence that it plays a role in antigen cross presentation, although it is capable of mediating internalization of Grp94 and calreticulin (Berwin et al., 2003; Wang et al., 2007). SRECI and LOX-1 each appeared to mediate approximately $50 \%$ of the cross presentation of the ovalbumin (Ova) SIINFEKL epitope in murine BMDC (Murshid et al., 2010).

It has recently been shown that GRP94 can lead to antigen cross presentation in a receptor-independent manner, questioning the significance of HSP receptors (Jockheck-Clark et al., 2010). This issue was also recently examined by Murshid et al. (2010) who tested the ability of $\mathrm{CHO}$ cells to cross prime B3Z T cell hybridoma cells that respond to Ova peptide SIINFEKL in the context of $\mathrm{H} 2 \mathrm{~kb}$. Wild-type $\mathrm{CHO}$ cells or $\mathrm{CHO}$ engineered to express $\mathrm{H} 2 \mathrm{~kb}$ on the surface and exposed to Hsp90-Ova or Hsp90-associated with N-terminally extended SIINFEKL failed to cross prime B3Z cells. However, co-expression of $\mathrm{H} 2 \mathrm{~kb}$ and the receptor SCRECI rendered the $\mathrm{CHO}$ cells competent to cross prime the $\mathrm{B} 3 \mathrm{Z}$ cells (Murshid et al., 2010). These studies strongly suggest a role for receptors such as SR in cross presentation of HSP-bound antigens, although they cannot rule out a role for non-receptor mechanisms in some cell types (Jockheck-Clark et al., 2010).

The SR were initially shown to be important in HSP binding and CTL activation using a wide range of SR agonists. Compounds that bind SR such as such as maleylated BSA, Oxidized LDL, acetylated BSA, poly-inosine, and fucoidan all reduce Hsp70 binding to DC (Delneste et al., 2002). Class E SR family member LOX-1 was shown to be expressed at high levels in myeloid derived DC and in mouse bone marrow derived DC and it was demonstrated conclusively that this receptor is involved in antigen cross presentation by DC exposed to Hsp70-antigen complexes (Delneste et al., 2002). Indeed, anti-LOX-1 antibodies coupled to Ova could mediate the rejection of Ova expressing tumor cells. Recently, it was also shown that LOX-1 is an Hsp60 receptor and Hsp60-fused Ova can be processed by DC and can be delivered to MHCI molecules to activate CD8 $+\mathrm{T}$ cells response, suggesting their role in antigen cross presentation (Xie et al., 2010).

Class F scavenger receptor SRECI binds avidly to Hsp70, Hsp90, Grp94, Hsp110, and Grp170 with or without associated antigens and appears to be a common receptor for HSPs in DC (Manjili et al., 2002; Berwin et al., 2004; Murshid et al., 2010). Hsp90antigen complexes can be bound and internalized in a range of cell types including DC and packaged into distinct classes of vesicles by SRECI, involving lipid raft localization of the receptor-HSP.PC complex and taken up in a dynamin- and clathrin-independent pathway (Murshid et al., 2010). HSP.PC along with SRECI is endocytosed into tubule like vesicles termed Clathrin-independent carriers (CLIC) or GPI anchored protein-enriched endocytic compartments (GEEC; Doherty and McMahon, 2009; Gupta et al., 2009). Although the significance of entry of HSP-SRECI complexes through the CLIC/GEEC pathway is not entirely clear, it does permit regulation of antigen cross presentation by signal transducing molecules as discussed below. In addition, LOX-1 is internalized through a dynamin-dependent mechanism involving association with caveolae, distinct from the GEEC pathway used by SRECI but is also able to mediate cross presentation of peptides bound to HSPs (Murphy et al., 2008).

It is not clear to what degree the antigen presentation pathways utilized by HSP-chaperoned antigens after endocytosis conform to those used by unchaperoned antigens. For instance, particulate antigen processing toward the class I pathway, involves an elevation in intraphagosomal $\mathrm{pH}$ and it is not yet known if such processes are important for HSP-mediated antigen processing and presentation (Inaba and Inaba, 2005). Intravesicular $\mathrm{pH}$ changes could be involved in the dissociation of peptides from the HSP, as well as subsequent loading onto MHC class I. Cross presentation in BMDC after Hsp90-Ova peptide exposure was reduced by exposure to chloroquine, an inhibitor of vesicular acidification suggesting that low $\mathrm{pH}$ may be required at least for cross presentation through the vesicular pathway (Murshid et al., 2010). So far it has been established that both the endosome-plasma membrane and endosome to cytosol-cell membrane mechanisms are involved in Hsp70 and Hsp90-mediated antigen cross presentation in BMDC (Murshid et al., 2010). Partition of antigens between these two pathways may depend on the size of the client polypeptide associated with the HSP. Smaller, Ova-derived peptides bound to Hsp90 could be efficiently cross presented through a mechanism resembling the vesicular pathway and such processing was blocked by cysteine protease inhibitors and, primaquine an antagonist of membrane recycling (Murshid et al., 2010). Larger polypeptides associated with Hsp90, such as full length ovalbumin required TAP expression and proteasome function, indicating a key role for the endosome-cytosol pathway in processing these larger antigens (Murshid et al., 2010). HSP chaperoning of peptide antigens may increase the efficiency of antigen processing by protecting peptides from proteolysis during trafficking through the cell compartments and reducing the amounts of antigen required to initiate CD8+ T cell cross priming (Kunisawa and Shastri, 2006). One question that is currently unclear is how far along the journey of antigen cross presentation the HSP-receptor-polypeptide complex remains intact. Hsp90, SRECI, and Ova-derived peptides have been detected co-localizing in endosomal compartments of BMDC (Murshid et al., 2010). In addition, in DC incubated with Hsp90-Ova complexes, internalized Hsp90 and Ova were shown to co-localize with cytosolic proteasomes (Oura et al., 2011). Localization of Ova to proteasomes and TAP-dependent antigen cross presentation could be abolished by introduction of anti-Hsp90 antibodies into cells, indicating a key role for Hsp90 in targeting associated Ova molecules to proteasomes (Oura et al., 2011). It has been shown recently that intracellular Hsp90 plays an essential role in antigen cross presentation by permitting the transport of model antigen Ova out of endosomes and into the cytosol to permit processing by the proteasome (Ichiyanagi et al., 2010; 
Imai et al., 2011). By contrast with free Ova, cross presentation of Hsp90-chaperoned Ova appeared to be independent of endogenous Hsp90, suggesting that exogenous Hsp90 may facilitate antigen transport across the endosomal membrane independently of the endogenous chaperone (Oura et al., 2011). Ultimately, peptides dissociate from the HSP prior to processing and presentation and receptors such as SREC1 are likely recycled to the cell surface. Fluorescently labeled Hsp90 was observed for approximately $60 \mathrm{~min}$ in murine BMDC, after binding and internalization, before fluorescence became undetectable (Murshid, A., unpublished data). Hsp90 taken up from the extracellular medium and entering endosomes may be broken down by intravesicular proteases or lysosomal hydrolases.

\section{ROLE OF INTRACELLULAR MOLECULAR CHAPERONES IN CROSS PRESENTATION OF ANTIGENS IN CELLULAR MATERIALS ENGULFED BY APC}

Tumor cells or virally infected cells may trigger immunity either directly when MHC class I antigen complexes on the surface interact with CD8+ $\mathrm{T}$ cells or when such cells die, are engulfed by APC and epitopes are cross presented to the CD8+ cells (Wolkers et al., 2001). There has been some suggestion that HSPs might play a role in the second process, in cross presentation of antigens sequestered by molecular chaperones by APC after engulfment of cell bodies. HSP-chaperoned peptides appear to exist in detectable quantities in cells and could be significant in this latter process. Indeed it was suggested by Binder et al. (2000) that essentially all antigenic epitopes in cells may be bound to molecular chaperones (Binder and Srivastava, 2005). However in a study of Ova epitope presentation in cell lysates, free unprocessed proteins appeared to be the dominant form of antigens for cross priming, far exceeding the activities of free or HSP-chaperoned peptides (Shen and Rock, 2004). Most peptides generated by proteolysis of cytoplasmic proteins have a very short half-life, surviving for only a few seconds, although a minority can survive for longer periods by association with TAP (Reits et al., 2003). There has been some suggestion that HSPs could chaperone such peptides in the cytoplasm and protect them from cytoplasmic aminopeptidases (Srivastava, 2002). Intracellular chaperones may acquire peptide antigens during endogenous antigen processing (Kunisawa and Shastri, 2003, 2006). Indeed Shastri et al. have shown that Hsp90a is essential in stabilizing large peptide intermediates generated by the proteasome from Ova and for presentation through the MHC class I pathway (Kunisawa and Shastri, 2006). In addition, a minority of viral epitopes that can bind Hsp90 $\alpha$ appear to be stabilized and are important in cross priming (Lev et al., 2008). However the exact place of HSPs in cytoplasmic antigen processing is uncertain. When peptides are taken up by TAP into the ER they could potentially encounter other molecular chaperones, including the Hsp70 paralog Grp78 and the Hsp90 paralog Grp94, molecules with suspected immune functions. It is not clear whether Grp78 binds peptides or participates in antigen presentation although it has known immune functions in the chaperoning of IgG (Kozutsumi et al., 1989). In addition, Grp94 does not appear to participate in cross priming of anti-viral CD8+ cells in vivo (Nicchitta, 2003; Lev et al., 2009). Another ER chaperone shown in vitro to bind avidly to polypeptide clients is Grp170 (Park et al., 2006). However it is not clear whether ER resident Grp170 can acquire peptide antigens in vivo or can play a role in antigen presentation, although its high avidity for peptides and proteins suggests investigation of such a possibility.

\section{RELEASE OF HSPS FROM CELLS INTO THE EXTRACELLULAR ENVIRONMENT}

Heat shock proteins can also be released from cells, tissues, and access the extracellular environment, although their functions outside the cell are largely unknown and much debated (Pockley et al., 2008). HSPs can be released from tumor cells or macrophages into the extracellular microenvironment through mechanisms involving secretory lysosomes, very similar to the processes used by cells to release interleukin-1 $\beta$ (Mambula and Calderwood, 2006b). This pathway of non-canonical HSP secretion occurs at a gradual basal rate and can be triggered in cells by stresses such as fever range heat or by exposing macrophages to live E. coli bacteria (Mambula and Calderwood, 2006b; Mambula, S. S., Murshid, A., and Calderwood, S. K., in preparation). HSPs released in this way could potentially export antigenic peptides acquired during intracellular antigen processing as cargo, interact with APC and trigger cross priming of CD8+ subsets. However, it is not clear how stable antigen binding would be under these circumstances. It is known that for hspa family chaperones such as Hsp70, peptide binding is relatively stable when ADP rather than ATP is bound to the chaperone. Hsp70 is also released from necrotic cells and under these circumstances might be thought to bind associated polypeptides, as intracellular ATP levels would decline rapidly in necrosis and the chaperone, in its ADP-bound form would bind peptides more effectively (Mambula and Calderwood, 2006a). Hsp70 released from cells undergoing necrotic death has been shown to lead to a strong $\mathrm{T}$ cell mediated anti-tumor immune response (Daniels et al., 2004). In addition, it was shown that Grp170, engineered for secretion into the extracellular environment initiated tumor rejection due to activation of $\mathrm{NK}$ and $\mathrm{CD} 8+$ cells, indicating the potential of this approach (Wang et al., 2003).

In addition to secretion in free form, HSPs have been shown to be released from cells after being packaged in exosomes. Exosomes derived from multivesicular bodies in DC contain Hsp70 and are known to play a major role in cross priming of $\mathrm{T}$ lymphocytes (Chaput et al., 2006). Thus Hsp70 in exosomes could potentially play a significant role in immunity. However, exosomes from tumors (EL4 Thymoma) which contain Hsp70 on the vesicular surface were shown to be immunosuppressive through interaction with myeloid derived suppressor cells (MDSC; Chalmin et al., 2010). Relatively little work has been carried out in this interesting area and we anticipate further studies.

\section{EXTRACELLULAR HSPS AND THE CLASS II PATHWAY}

Cross presentation of HSP-associated antigens, although essential would not be sufficient to fully program CTL and in the absence of other signals, CTL would ultimately fail to actively proliferate and could undergo programmed cell death. For a sturdy CTL response, DC would process and present antigens via the MHC class II pathway and thus activate CD4 $+\mathrm{T}$ helper cells. It has 
been shown that HSPs can chaperone external antigens through the MHC class II pathway and activate CD4+ T cells (Gong et al., 2009). As mentioned above, unchaperoned antigens, after uptake into phagosomes, traverse separate trafficking routes in DC that direct them to compartments in which association with MHC class I or II molecules can occur. It is not clear which mechanisms are involved in the partitioning of HSP-chaperoned antigens into these pathways. We have shown however that SRECI is involved in HSP-mediated CD4+ T cell activation and our preliminary studies indicate that the early endocytic and regulatory stages of this process, such as uptake of HSP.PC through SRECI, involve similar pathways to those in Class I presentation (Gong et al., 2009; Murshid, A., and Calderwood, S. K., in preparation). Activation of CTL is thought to require DC licensing, in which an individual DC interacts with both CD4+ and CD8+ T cells (Bennett et al., 1997, 1998). Initial interaction of CD40 ligand on the CD4+ $\mathrm{T}$ cell surface with CD40 on the DC sets in motion a series of events including the induction of co-stimulatory molecules such as CD80 (B7-1), CD86 (B7-2) that permit subsequent CD8+ T cells activity (Kurts et al., 2010; Figure 1). Co-stimulatory molecules are recognized by counter receptors such as CD28 expressed on $\mathrm{T}$ cells and reinforce the signals induced in CD8 $+\mathrm{T}$ cells by MHCI-peptide complex-T cell receptor binding (van der Merwe et al., 1997; Rudd et al., 2009; Figure 1). Most HSPs are likely to bind to a wide spectrum of intracellular antigens, containing both MHC class I and class II epitopes and may favor the reactions involved in DC licensing.

\section{HSP ASSOCIATION WITH PATTERN RECOGNITION RECEPTORS}

Cytotoxic lymphocytes activation can also be reinforced by signals induced by pathogen-associated molecular patterns (PAMPs). During infection, PAMPs bind to several classes of pattern recognition receptors (PRR) such as such as Toll-like receptors (TLR) and trigger innate immune responses in APC (Yamamoto and Takeda, 2010). This type of signaling activates a number of transcription factors, most notably nuclear factor $-\kappa B(\mathrm{NF} \kappa \mathrm{B})$ that can stimulate transcription of genes encoding inflammatory cytokines and co-stimulatory molecules such as B7-1 and B7-2 (Parra et al., 1995; Rudd et al., 2009). Innate immune activation through these pathways can thus reinforce CTL programming through pathways overlapping those induced by CD40L-CD40 interactions between CD4+ T cells and DC (Maurer et al., 2002; Schulz et al., 2005; Najar and Dutz, 2007; Figure 1). HSPs may potentially function as DAMPs (damage associated molecular patterns) and induce innate immunity under sterile conditions through interaction with PRR (Chase et al., 2007; Wheeler et al., 2009). Indeed, there is an evidence for HSPs interacting with PRR such as TLR2 and TLR4 (Gong et al., 2009; Figure 1). It was shown that SRECIdependent tumor immunity induced by $\mathrm{Hsp} 70$ vaccines requires TLR signaling and knockout of the TLR signaling intermediate Myd88 or the receptors TLR2 and TLR4 obliterates tumor immunity (Gong et al., 2009). In addition to affecting innate immune transcription, TLR signaling also mediates a number of stages in antigen cross presentation and positive HSP interaction with TLRs may thus reinforce antigen cross presentation (Amigorena and Savina, 2010). As CTL programming involves the interaction of three rare cell types, including DC, CD8 $+\mathrm{T}$ cells, and CD4+ T cells, this confluence may be strongly influenced by the release of inflammatory CCL cytokines from DC. It may be notable that Hsp70 has been shown to trigger release of CCL cytokines in a TLR4 dependent manner and that mycobacterial Hsp70 binds directly to CCL receptor CCR5 (Whittall et al., 2006; Chen et al., 2009).

\section{HSP-SCAVENGER RECEPTOR INTERACTIONS AND TRANSMEMBRANE SIGNALING}

In addition to internalizing bound peptides, ligand-bound HSPs may play significant roles in cell signaling pathways that impact immunity. LOX-1 and SRECI can both interact with TLR2 to induce innate immune signaling and transcription (Jeannin et al., 2005). Although little is known regarding HSP-LOX-1 signaling, binding of LOX-1 to other ligands, such as modified LDL induces MAP kinase activity, NF- $\kappa \mathrm{B}$, and pro-inflammatory signaling through the small intracellular domain of the receptor (Chen et al., 2002). However, when HSPs interact with class I scavenger receptor SRA/CD204, innate immune signaling through TLR4, NFKB, and MAP kinase is reduced, and CD4+ T cell activation is inhibited (Yi et al., 2011). Knockout of the SRA/CD204 gene, although decreasing Hsp110 binding to DC led to a profoundly increased ability to stimulate melanoma antigen gp100-specific naïve T cells, compared to wild-type mice (Qian et al., 2011). HSPSR interactions may thus send both pro-immune and inhibitory signals resulting in a tightly regulated system. Signaling through SRECI appears to reinforce antigen cross presentation and the appearance of SRECI in lipid rafts after binding Hsp90 may be a key stage in this process (Murshid et al., 2010). Lipid rafts are cholesterol- and sphingolipid-rich membrane microdomains that can concentrate molecules involved in cell signaling (Lingwood et al., 2009). Although lacking the glycerophosphoinositide anchor domain motifs found in many raft-associated membrane proteins, SRECI contains other motifs that would permit it to associate with lipid rafts (Murshid et al., 2011a). Indeed, Hsp90-SRECI complexes associate with the small GTPase Cdc42 and non-receptor tyrosine kinase Src, molecules tightly associated with lipid rafts (Murshid et al., 2010). These associations appear to be important in regulating antigen cross presentation of $\mathrm{Hsp} 90$-associated antigens in DC.

Lipid rafts also concentrate intermediates in the TLR4 signaling pathway in response to innate immune stimuli (Triantafilou et al., 2002). Indeed, preliminary studies show significant colocalization of Hsp90-SRECI complexes with TLR4 (Murshid, A., and Calderwood, S. K., in preparation). HSP-triggered signaling through SRECI and LOX-1 may thus be involved both in amplifying antigen cross presentation and in stimulating innate immunity and these receptors may operate in concert to thwart the inhibitory effects of HSP-SRA/CD204 signaling. It may be significant that LOX-1, with essentially no sequence similarity to SRECI associates with TLR2 after ligand binding and mediates immune responses in a similar way to SRECI (Jeannin et al., 2005). The similarity in immune properties and protein interaction partners between SRECI and LOX-1 despite sequence homology may suggest functional interaction on the cell surface after HSP binding. 


\section{CONCLUSION}

On theoretical grounds, HSPs might be seen as ideal sources of tumor antigens due to their high abundance in cancer and their molecular chaperone functions. However, the nature of the polypeptides chaperoned by HSPs which carry the "antigenic load" of the HSP.PC is less clear. Most cytoplasmic peptides appear to be in free, unchaperoned form unless acquired by TAP, although Hsp90 appears to be an exception to this rule and appears to be capable of binding to peptides exiting the proteasome. However, Hsp70 and Hsp90 both associate with intact proteins in vivo during protein folding and quality control, and the antigenic nature of extracellular HSPs extracted from tumors may thus at least partially reflect stable chaperone binding to antigenic proteins.

It seems clear that HSPs can mediate cross presentation of acquired tumor antigens by APC. This process may involve receptor-mediated uptake by SR LOX-1 or SRECI or receptorindependent uptake. If small peptides are bound to HSPs they can then be presented to CD8+ cells through the rapid vacuolar pathway of antigen cross presentation. Hsp90 appears to be able to chaperone whole proteins such as ovalbumin across the plasma membrane, into endosomes, and direct them to the proteasome for processing and presentation by MHC class I molecules.

In addition to directing antigens toward the Class I pathway, HSPs can chaperone antigens toward the Class II pathway of antigen presentation. This effect may be significant in recruiting

\section{REFERENCES}

Ackerman, A. L., Giodini, A., and Cresswell, P. (2006). A role for the endoplasmic reticulum protein retrotranslocation machinery during crosspresentation by dendritic cells. Immunity 25, 607-617.

Ackerman, A. L., Kyritsis, C., Tampe, R., and Cresswell, P. (2003). Early phagosomes in dendritic cells form a cellular compartment sufficient for cross presentation of exogenous antigens. Proc. Natl. Acad. Sci. U.S.A. 100, 12889-12894.

Amigorena, S., and Savina, A. (2010). Intracellular mechanisms of antigen cross presentation in dendritic cells. Curr. Opin. Immunol. 22, 109-117.

Bennett, S. R., Carbone, F. R., Karamalis, F., Flavell, R. A., Miller, J. F., and Heath, W. R. (1998). Help for cytotoxic-T-cell responses is mediated by CD40 signalling. Nature 393, 478-480.

Bennett, S. R., Carbone, F. R., Karamalis, F., Miller, J. F., and Heath, W. R. (1997). Induction of a CD8+ cytotoxic $\mathrm{T}$ lymphocyte response by cross-priming requires cognate CD4+ T cell help. J. Exp. Med. 186, 65-70.

Berwin, B., Delneste, Y., Lovingood, R. V., Post, S. R., and Pizzo, S. V. (2004). SREC-I, a type F scavenger receptor, is an endocytic receptor antigen-specific Th1 helper cells to mediate DC licensing and amplify CTL processing.

Association of HSP-antigen complexes with SR SRECI and LOX-1 may help to amplify cross presentation of antigens by triggering signal transduction pathways emanating from lipid rafts. HSP-bound SR can associate with lipid rafts and activate signaling through small GTPase $\mathrm{Cdc} 42$, tyrosine kinase c-sec, and induce activity of PRR such as TLR2. There also appear to be HSP-mediated regulatory signals and binding to SRA/CD204 can inhibit immunity.

There remains a need to identify a number of unresolved cell biological and biochemical aspects of HSP.PC-mediated immunity. These include further investigation of the signals initiated when HSPs bind to cell surface receptors, the kinetics of compartmentalization of the chaperoned peptides and the routes of trafficking and processing of peptides after release from HSP. Advanced technology studies of the trafficking of single cell molecules may open up this field. Elucidating the mechanisms of antigen cross presentation of HSP-bound antigens via MHCI and the signaling events associated with this immunogenic effect may allow development of superior HSP-based vaccines and immunotherapy protocols.

\section{ACKNOWLEDGMENTS}

This work was supported by NIH research grants RO-1CA047407, R01CA119045, and RO-1CA094397. for calreticulin. J. Biol. Chem. 279, 51250-51257.

Berwin, B., Hart, J. P., Rice, S., Gass, C., Pizzo, S. V., Post, S. R., and Nicchitta, C. V. (2003). Scavenger receptor-A mediates gp96/GRP94 and calreticulin internalization by antigen-presenting cells. $E M B O \mathrm{~J}$. 22, 6127-6136.

Binder, R. J., Han, D. K., and Srivastava, P. K. (2000). CD91: a receptor for heat shock protein gp96. Nat. Immunol. 1, 151-155.

Binder, R. J., and Srivastava, P. K. (2005). Peptides chaperoned by heat-shock proteins are a necessary and sufficient source of antigen in the crosspriming of CD8+ T cells. Nat. Immunol. 6, 593-599.

Bonifaz, L., Bonnyay, D., Mahnke, K., Rivera, M., Nussenzweig, M. C., and Steinman, R. M. (2002). Efficient targeting of protein antigen to the dendritic cell receptor DEC-205 in the steady state leads to antigen presentation on major histocompatibility complex class I products and peripheral CD8+ $\mathrm{T}$ cell tolerance. J. Exp. Med. 196, 1627-1638.

Burgdorf, S., Lukacs-Kornek, V., and Kurts, C. (2006). The mannose receptor mediates uptake of soluble but not of cell-associated antigen for cross-presentation. J. Immunol. 176, 6770-6776.
Burgdorf, S., Schuette, V., Semmling, V., Hochheiser, K., Lukacs-Kornek, V. Knolle, P. A., and Kurts, C. (2010). Steady-state cross-presentation of OVA is mannose receptordependent but inhibitable by collagen fragments. Proc. Natl. Acad. Sci. U.S.A. 107, E48-E49.

Calderwood, S. K., and Gong, J. (2011). Molecular chaperones in mammary cancer growth and breast tumor therapy. J. Cell. Biochem. doi: 10.002/jcb.23461. [Epub ahead of print].

Calderwood, S. K., Khaleque, M. A., Sawyer, D. B., and Ciocca, D. R. (2006). Heat shock proteins in cancer: chaperones of tumorigenesis. Trends Biochem. Sci. 31, 164-172.

Calderwood, S. K., Xie, Y., Wang, X., Khaleque, M. A., Chou, S. D., Murshid, A., Prince, T., and Zhang, Y. (2010). Signal transduction pathways leading to heat shock transcription. Sign. Transduct. Insights 2 , 13-24.

Carter, H., Chen, S., Isik, L., Tyekucheva, S., Velculescu, V. E., Kinzler, K. W., Vogelstein, B., and Karchin, R. (2009). Cancer-specific highthroughput annotation of somatic mutations, computational prediction of driver missense mutations. Cancer Res. 69, 6660-6667.
Chalmin, F., Ladoire, S., Mignot, G., Vincent, J., Bruchard, M., RemyMartin, J. P., Boireau, W., Rouleau, A., Simon, B., Lanneau, D., De Thonel, A., Multhoff, G., Hamman, A., Martin, F., Chauffert, B., Solary, E., Zitvogel, L., Garrido, C., Ryffel, B., Borg, C., Apetoh, L., Rébé, C., and Ghiringhelli, F. (2010). Membrane-associated Hsp72 from tumor-derived exosomes mediates STAT3-dependent immunosuppressive function of mouse and human myeloid-derived suppressor cells. $J$. Clin. Invest. 120, 457-471.

Chandawarkar, R. Y., Wagh, M. S., Kovalchin, J. T., and Srivastava, P. (2004). Immune modulation with high-dose heat-shock protein gp96: therapy of murine autoimmune diabetes and encephalomyelitis. Int. Immunol. 16, 615-624.

Chandawarkar, R. Y., Wagh, M. S., and Srivastava, P. K. (1999). The dual nature of specific immunological activity of tumor-derived gp96 preparations. J. Exp. Med. 189, 1437-1442.

Chaput, N., Flament, C., Viaud, S., Taieb, J., Roux, S., Spatz, A., André, F., LePecq, J. B., Boussac, M., Garin, J., Amigorena, S., Théry, C., and Zitvogel, L. (2006). Dendritic cell derived-exosomes, biology and clinical implementations. J. Leukoc. Biol. $80,471-478$. 
Chase, M. A., Wheeler, D. S., Lierl, K. M., Hughes, V. S., Wong, H. R., and Page, K. (2007). Hsp72 induces inflammation and regulates cytokine production in airway epithelium through a TLR4- and NF-kappaB-dependent mechanism. J. Immunol. 179, 6318-6324.

Chen, M., Masaki, T., and Sawamura, T. (2002). LOX-1, the receptor for oxidized low-density lipoprotein identified from endothelial cells, implications in endothelial dysfunction and atherosclerosis. Pharmacol. Ther. 95, 89-100.

Chen, T., Guo, J., Han, C., Yang, M., and Cao, X. (2009). Heat shock protein 70 , released from heatstressed tumor cells, initiates antitumor immunity by inducing tumor cell chemokine production and activating dendritic cells via TLR4 pathway. J. Immunol. 182, 1449-1459.

Craig, E. A. (1985). The heat shock response. CRC Crit. Rev. Biochem. $18,239-280$

Cresswell, P. (1994). Assembly, transport, and function of MHC class II molecules. Annu. Rev. Immunol. 12, 259-293.

Cresswell, P., Ackerman, A. L., Giodini, A., Peaper, D. R., and Wearsch, P. A. (2005). Mechanisms of MHC class I-restricted antigen processing and cross-presentation. Immunol. Rev. 207, 145-157.

Daniels, G. A., Sanchez-Perez, L., Diaz, R. M., Kottke, T., Thompson, J., Lai, M., Gough, M., Karim, M., Bushell, A., Chong, H., Melcher, A., Harrington, K., and Vile, R. G. (2004). A simple method to cure established tumors by inflammatory killing of normal cells. Nat. Biotechnol. 22, 1125-1132.

Delneste, Y., Magistrelli, G., Gauchat, J., Haeuw, J., Aubry, J., Nakamura, K., Kawakami-Honda, N., Goetsch, L., Sawamura, T., Bonnefoy, J., and Jeannin, P. (2002). Involvement of LOX-1 in dendritic cell-mediated antigen cross-presentation. Immunity 17, 353-362.

Doherty, G. J., and McMahon, H. T. (2009). Mechanisms of endocytosis. Annu. Rev. Biochem. 78, 857-902.

Ellis, R. J. (2007). Protein misassembly: macromolecular crowding and molecular chaperones. Adv. Exp. Med. Biol. 594, 1-13.

Engelhard, V. H., Bullock, T. N., Colella, T. A., Sheasley, S. L., and Mullins, D. W. (2002). Antigens derived from melanocyte differentiation proteins: self-tolerance, autoimmunity, and use for cancer immunotherapy. Immunol. Rev. 188, 136-146.
Enomoto, Y., Bharti, A., Khaleque, A. A., Song, B., Liu, C., Apostolopoulos, V., Xing, P. X., Calderwood, S. K., and Gong, J. (2006). Enhanced immunogenicity of heat shock protein 70 peptide complexes from dendritic cell-tumor fusion cells. J. Immunol. 177, 5946-5955.

Flynn, G. C., Chappell, T. G., and Rothman, J. E. (1989). Peptide binding and release by proteins implicated as catalysts of protein assembly. Science $245,385-390$.

Fourie, A. M., Sambrook, J. F., and Gething, M. J. (1994). Common and divergent peptide binding specificities of hsp70 molecular chaperones. J. Biol. Chem. 269, 30470-30478.

Geijtenbeek, T. B., and Gringhuis, S. I. (2009). Signalling through C-type lectin receptors: shaping immune responses. Nat. Rev. Immunol. 9, 465-479.

Gong, J., Zhang, Y., Durfee, J., Weng, D., Liu, C., Koido, S., Song, B., Apostolopoulos, V., and Calderwood, S. K. (2010). A heat shock protein 70-based vaccine with enhanced immunogenicity for clinical use. $J$. Immunol. 184, 488-496.

Gong, J., Zhu, B., Murshid, A., Adachi, H., Song, B., Lee, A., Liu, C., and Calderwood, S. K. (2009). T cell activation by heat shock protein 70 vaccine requires TLR signaling and scavenger receptor expressed by endothelial cells-1. J. Immunol. 183, 3092-3098.

Gragerov, A., and Gottesman, M. E. (1994). Different peptide binding specificities of hsp70 family members. J. Mol. Biol. 241, 133-135.

Grossmann, M. E., Madden, B. J., Gao, F., Pang, Y. P., Carpenter, J. E., McCormick, D., and Young, C. Y. (2004). Proteomics shows Hsp70 does not bind peptide sequences indiscriminately in vivo. Exp. Cell Res. 297, 108-117.

Gupta, G. D., Swetha, M. G., Kumari, S., Lakshminarayan, R., Dey, G., and Mayor, S. (2009). Analysis of endocytic pathways in Drosophila cells reveals a conserved role for GBF1 in internalization via GEECs. PLoS ONE 4, e6768. doi:10.1371/journal.pone. 0006768

Hanahan, D., and Weinberg, R. A. (2000). The hallmarks of cancer. Cell 100, 57-70.

Hanahan, D., and Weinberg, R. A. (2011). Hallmarks of cancer: the next generation. Cell 144, 646-674.

Heath, W. R., and Carbone, F. R. (2009). Dendritic cell subsets in primary and secondary $\mathrm{T}$ cell responses at body surfaces. Nat. Immunol. 10, 1237-1244.
Ichiyanagi, T., Imai, T., Kajiwara, C. Mizukami, S., Nakai, A., Nakayama, T., and Udono, H. (2010). Essential role of endogenous heat shock protein 90 of dendritic cells in antigen cross-presentation. J. Immunol. 185, 2693-2700.

Idoyaga, J., Lubkin, A., Fiorese, C., Lahoud, M. H., Caminschi, I., Huang, Y., Rodriguez, A., Clausen, B. E., Park, C. G., Trumpfheller, C., and Steinman, R. M. (2011). Comparable Thelper 1 (Th1) and CD8 T-cell immunity by targeting HIV gag p24 to CD8 dendritic cells within antibodies to Langerin, DEC205, and Clec9A. Proc. Natl. Acad. Sci. U.S.A. 108, 2384-2389.

Imai, T., Kato, Y., Kajiwara, C. Mizukami, S., Ishige, I., Ichiyanagi, T., Hikida, M., Wang, J. Y., and Udono, H. (2011). Heat shock protein 90 (HSP90) contributes to cytosolic translocation of extracellular antigen for cross-presentation by dendritic cells. Proc. Natl. Acad. Sci. U.S.A. 108, 16363-16368.

Inaba, K., and Inaba, M. (2005). Antigen recognition and presentation by dendritic cells. Int. J. Hematol. 81, 181-187.

Jeannin, P., Bottazzi, B., Sironi, M., Doni, A., Rusnati, M., Presta, M., Maina, V., Magistrelli, G., Haeuw, J. F., Hoeffel, G., Thieblemont, N., Corvaia N., Garlanda, C., Delneste, Y., and Mantovani, A. (2005). Complexity and complementarity of outer membrane protein A recognition by cellular and humoral innate immunity receptors. Immunity 22, 551-560.

Jockheck-Clark, A. R., Bowers, E. V. Totonchy, M. B., Neubauer, J., Pizzo, S. V., and Nicchitta, C. V. (2010). Re-examination of CD91 function in GRP94 (glycoprotein 96) surface binding, uptake, and peptide cross-presentation. J. Immunol. 185 6819-6830.

Kamal, A., Thao, L., Sensintaffar, J., Zhang, L., Boehm, M. F., Fritz, L. C., and Burrows, F. J. (2003). A highaffinity conformation of $\mathrm{Hsp} 90$ confers tumour selectivity on $\mathrm{Hsp} 90$ inhibitors. Nature 425, 407-410.

Kozutsumi, Y., Normington, K., Press, E., Slaughter, C., Sambrook, J., and Gething, M. J. (1989). Identification of immunoglobulin heavy chain binding protein as glucose-regulated protein 78 on the basis of amino acid sequence, immunological crossreactivity, and functional activity. $J$. Cell Sci. Suppl. 11, 115-137.

Kunisawa, J., and Shastri, N. (2003). The group II chaperonin TRiC protects proteolytic intermediates from degradation in the MHC class I antigen processing pathway. Mol. Cell 12, 565-576.

Kunisawa, J., and Shastri, N. (2006). Hsp90alpha chaperones large Cterminally extended proteolytic intermediates in the MHC class I antigen processing pathway. Immunity 24, 523-534.

Kurotaki, T., Tamura, Y., Ueda, G., Oura, J., Kutomi, G., Hirohashi, Y., Sahara, H., Torigoe, T., Hiratsuka, H., Sunakawa, H., Hirata, K., and Sato, N. (2007). Efficient crosspresentation by heat shock protein 90-peptide complex-loaded dendritic cells via an endosomal pathway. J. Immunol. 179, 1803-1813.

Kurts, C., Robinson, B. W., and Knolle, P. A. (2010). Cross-priming in health and disease. Nat. Rev. Immunol. 10, 403-414.

Lev, A., Dimberu, P., Das, S. R., Maynard, J. C., Nicchitta, C. V., Bennink, J. R., and Yewdell, J. W. (2009). Efficient cross-priming of antiviral CD8 + T cells by antigen donor cells is GRP94 independent. J. Immunol. 183, 4205-4210.

Lev, A., Takeda, K., Zanker, D., Maynard, J. C., Dimberu, P., Waffarn, E., Gibbs, J., Netzer, N., Princiotta, M. F., Neckers, L., Picard, D., Nicchitta, C. V., Chen, W., Reiter, Y., Bennink, J. R., and Yewdell, J. W. (2008). The exception that reinforces the rule: crosspriming by cytosolic peptides that escape degradation. Immunity 28, 787-798.

Lingwood, D., Kaiser, H. J., Levental, I., and Simons, K. (2009). Lipid rafts as functional heterogeneity in cell membranes. Biochem. Soc. Trans. 37(Pt 5), 955-960.

Mambula, S. S., and Calderwood, S. K. (2006a). Heat induced release of Hsp70 from prostate carcinoma cells involves both active secretion and passive release from necrotic cells. Int. J. Hyperthermia 22, 575-585.

Mambula, S. S., and Calderwood, S. K. (2006b). Heat shock protein 70 is secreted from tumor cells by a nonclassical pathway involving lysosomal endosomes. J. Immunol. 177, 7849-7857.

Manjili, M. H., Henderson, R., Wang, X. Y., Chen, X., Li, Y., Repasky, E., Kazim, L., and Subjeck, J. R. (2002). Development of a recombinant HSP110-HER-2/neu vaccine using the chaperoning properties of HSP110. Cancer Res. 62, 1737-1742.

Manjili, M. H., Wang, X. Y., Chen, X., Martin, T., Repasky, E. A., Henderson, R., and Subjeck, J. R. (2003). HSP110-HER2/neu chaperone complex vaccine induces protective immunity against spontaneous 
mammary tumors in HER-2/neu transgenic mice. J. Immunol. 171, 4054-4061.

Maurer, T., Heit, A., Hochrein, H., Ampenberger, F., O’Keeffe, M., Bauer, S., Lipford, G. B., Vabulas, R. M., and Wagner, H. (2002). CpG-DNA aided cross-presentation of soluble antigens by dendritic cells. Eur. J. Immunol. 32, 2356-2364.

Mayer, M. P., and Bukau, B. (2005). Hsp70 chaperones: cellular functions and molecular mechanism. Cell. Mol. Life Sci. 62, 670-684.

Murphy, J. E., Vohra, R. S., Dunn, S., Holloway, Z. G., Monaco, A. P., Homer-Vanniasinkam, S., Walker, J. H., and Ponnambalam, S. (2008). Oxidised LDL internalisation by the LOX-1 scavenger receptor is dependent on a novel cytoplasmic motif and is regulated by dynamin-2. J. Cell. Sci. 121(Pt 13), 2136-2147.

Murshid, A., Gong, J., and Calderwood, S. K. (2010). Heat shock protein 90 mediates efficient antigen cross presentation through the scavenger receptor expressed by endothelial cells-I. J. Immunol. 185, 2903-2917.

Murshid, A., Gong, J., and Calderwood, S. K. (2011a). "Molecular chaperone receptors: binding and trafficking of molecular chaperones by class $\mathrm{F}$ and class G scavenger receptors," in Cellular Trafficking of Molecular Chaperones in Health and Disease, eds B. Henderson and A. G. Pockley. (in press).

Murshid, A., Theriault, J., Gong, J., and Calderwood, S. K. (2011b). Investigating receptors for extracellular heat shock proteins. Methods Mol. Biol. 787, 289-302.

Murshid, A., Gong, J., Stevenson, M. A., and Calderwood, S. K. (2011c). Heat shock proteins and cancer vaccines: developments in the past decade and chaperoning in the decade to come. Expert Rev. Vaccines 10, 1553-1568.

Najar, H. M., and Dutz, J. P. (2007). Topical TLR9 agonists induce more efficient cross-presentation of injected protein antigen than parenteral TLR9 agonists do. Eur. J. Immunol. 37, 2242-2256.

Neefjes, J. J., and Momburg, F. (1993). Cell biology of antigen presentation. Curr. Opin. Immunol. 5, 27-34.

Nicchitta, C. V. (2003). Re-evaluating the role of heat-shock proteinpeptide interactions in tumour immunity. Nat. Rev. Immunol. 3, 427-432.

Norbury, C. C., Basta, S., Donohue, K. B., Tscharke, D. C., Princiotta, M. F.,
Berglund, P., Gibbs, J., Bennink, J. R., and Yewdell, J. W. (2004). CD8+ $\mathrm{T}$ cell cross-priming via transfer of proteasome substrates. Science 304, 1318-1321.

Norbury, C. C., Malide, D., Gibbs, J. S., Bennink, J. R., and Yewdell, J. W. (2002). Visualizing priming of virusspecific CD8+ $\mathrm{T}$ cells by infected dendritic cells in vivo. Nat. Immunol. 3, 265-271.

Oh, H. J., Easton, D., Murawski, M., Kaneko, Y., and Subjeck, J. R. (1999). The chaperoning activity of hsp 110 . Identification of functional domains by use of targeted deletions. J. Biol. Chem. 274, 15712-15718.

Oura, J., Tamura, Y., Kamiguchi, K., Kutomi, G., Sahara, H., Torigoe, T., Himi, T., and Sato, N. (2011). Extracellular heat shock protein 90 plays a role in translocating chaperoned antigen from endosome to proteasome for generating antigenic peptide to be cross-presented by dendritic cells. Int. Immunol. 23, 223-237.

Park, J. E., Facciponte, J., Chen, X., MacDonald, I., Repasky, E. A., Manjili, M. H., Wang, X. Y., and Subjeck, J. R. (2006). Chaperoning function of stress protein grp170, a member of the hsp70 superfamily, is responsible for its immunoadjuvant activity. Cancer Res. 66, 1161-1168.

Parra, E., Varga, M., Sigvardsson, M., Hedlund, G., Kalland, T., Leanderson, T., Sjögren, H. O., and Dohlsten, M. (1995). Costimulation of human CD4+ T cells with LFA3 and B7 induce distinct effects on AP-1 and NF-kappa B transcription factors. J. Immunol. 155, 1132-1140.

Pockley, A. G., Muthana, M., and Calderwood, S. K. (2008). The dual immunoregulatory roles of stress proteins. Trends Biochem. Sci. 33, 71-79.

Qian, J., Yi, H., Guo, C., Yu, X., Zuo, D., Chen, X., Kane, J. M. III, Repasky, E. A., Subjeck, J. R., and Wang, X. Y. (2011). CD204 suppresses large heat shock protein-facilitated priming of tumor antigen gp100-specific $\mathrm{T}$ cells and chaperone vaccine activity against mouse melanoma. J. Immunol. 187, 2905-2914.

Reits, E., Griekspoor, A., Neijssen, J., Groothuis, T., Jalink, K., van Veelen, P., Janssen, H., Calafat, J., Drijfhout, J. W., and Neefjes, J. (2003). Peptide diffusion, protection, and degradation in nuclear and cytoplasmic compartments before antigen presentation by MHC class I. Immunity 18, 97-108.
Rock, K. L., Hearn, A., Chen, C. J., and Shi, Y. (2005). Natural endogenous adjuvants. Springer Semin. Immunopathol. 26, 231-246.

Rock, K. L., and Shen, L. (2005) Cross-presentation: underlying mechanisms and role in immune surveillance. Immunol. Rev. 207, 166-183.

Rudd, C. E., Taylor, A., and Schneider, H. (2009). CD28 and CTLA4 coreceptor expression and signal transduction. Immunol. Rev. 229, 12-26.

Scheibel, T., Weikl, T., and Buchner, J. (1998). Two chaperone sites in Hsp90 differing in substrate specificity and ATP dependence. Proc. Natl. Acad. Sci. U.S.A. 95, 1495-1499.

Schulz, O., Diebold, S. S., Chen, M. Naslund, T. I., Nolte, M. A., Alexopoulou, L., Azuma, Y. T., Flavell, R. A., Liljeström, P., and Reis e Sousa, C. (2005). Toll-like receptor 3 promotes cross-priming to virus-infected cells. Nature 433, 887-892.

Shen, L., and Rock, K. L. (2004). Cellular protein is the source of cross-priming antigen in vivo. Proc. Natl. Acad. Sci. U.S.A. 101, 3035-3040.

Srivastava, P. (2002). Interaction of heat shock proteins with peptides and antigen presenting cells: chaperoning of the innate and adaptive immune responses. Annu. Rev. Immunol. 20, 395-425.

Srivastava, P. K. (2000). Immunotherapy of human cancer, lessons from mice. Nat. Immunol. 1, 363-366.

Srivastava, P. K., and Old, L. J. (1988). Individually distinct transplantation antigens of chemically induced mouse tumors. Immunol. Today 9 , 78-83.

Steinman, R. M., Gutchinov, B., Witmer, M. D., and Nussenzweig, M. C. (1983). Dendritic cells are the principal stimulators of the primary mixed leukocyte reaction in mice. J. Exp. Med. 157, 613-627.

Tacken, P. J., Ginter, W., Berod, L., Cruz, L. J., Joosten, B., Sparwasser, T., Figdor, C. G., and Cambi, A. (2011). Targeting DC-SIGN via its neck region leads to prolonged antigen residence in early endosomes, delayed lysosomal degradation, and cross-presentation. Blood 118, 4111-4119.

Theriault, J. R., Adachi, H., and Calderwood, S. K. (2006). Role of scavenger receptors in the binding and internalization of heat shock protein 70. J. Immunol. 177, 8604-8611.
Triantafilou, M., Miyake, K., Golenbock, D. T., and Triantafilou, K. (2002). Mediators of innate immune recognition of bacteria concentrate in lipid rafts and facilitate lipopolysaccharide-induced cell activation. J. Cell. Sci. 115(Pt 12), 2603-2611.

Udono, H., and Srivastava, P. K. (1993). Heat shock protein 70-associated peptides elicit specific cancer immunity. J. Exp. Med. 178, 1391-1396.

van der Merwe, P. A., Bodian, D. L. Daenke, S., Linsley, P., and Davis, S. J. (1997). CD80 (B7-1) binds both CD28 and CTLA- 4 with a low affinity and very fast kinetics. J. Exp. Med. 185, 393-403.

Vogel, M., Mayer, M. P., and Bukau, B. (2006). Allosteric regulation of Hsp70 chaperones involves a conserved interdomain linker. J. Biol. Chem. 281, 38705-38711.

Vogelstein, B., and Kinzler, K. W. (1993). The multistep nature of cancer. Trends Genet. 9, 138-141.

Walters, J. J., and Berwin, B. (2005). Differential CD91 dependence for calreticulin and Pseudomonas exotoxin-A endocytosis. Traffic 6, 1173-1182.

Wang, X. Y., Facciponte, J., Chen, X., Subjeck, J. R., and Repasky, E. A. (2007). Scavenger receptor-A negatively regulates antitumor immunity. Cancer Res. 67, 4996-5002.

Wang, X. Y., Kazim, L., Repasky, E. A., and Subjeck, J. R. (2003). Immunization with tumor-derived ER chaperone grp170 elicits tumor-specific CD8+ T-cell responses and reduces pulmonary metastatic disease. Int. J. Cancer 105, 226-231.

Wang, X. Y., Sun, X., Chen, X., Facciponte, J., Repasky, E. A., Kane, J., and Subjeck, J. R. (2010). Superior antitumor response induced by large stress protein chaperoned protein antigen compared with peptide antigen. J. Immunol. 184, 6309-6319.

Wheeler, D. S., Chase, M. A., Senft, A. P., Poynter, S. E., Wong, H. R., and Page, K. (2009). Extracellular Hsp72, an endogenous DAMP, is released by virally infected airway epithelial cells and activates neutrophils via Tolllike receptor (TLR)-4. Respir. Res. 10,31 .

Whittall, T., Wang, Y., Younson, J., Kelly, C., Bergmeier, L., Peters, B., Singh, M., and Lehner, T. (2006). Interaction between the CCR5 chemokine receptors and microbial HSP70. Eur. J. Immunol. 36, 2304-2314.

Wolkers, M. C., Stoetter, G., VythDreese, F. A., and Schumacher, T. N. (2001). Redundancy of direct priming and cross-priming 
in tumor-specific CD8+ $\mathrm{T}$ cell responses. J. Immunol. 167, 3577-3584.

Xie, J., Zhu, H., Guo, L., Ruan, Y., Wang, L., Sun, L., Zhou, L., Wu, W., Yun, X., Shen, A., and Gu, J. (2010). Lectinlike oxidized low-density lipoprotein receptor-1 delivers heat shock protein 60 -fused antigen into the MHC class I presentation pathway. J. Immunol. 185, 2306-2313.

Yamamoto, M., and Takeda, K. (2010). Current views of toll-like receptor signaling pathways. Gastroenterol. Res. Pract. 2010, 240365.
Yi, H., Guo, C., Yu, X., Gao, P., Qian, J., Zuo, D., Manjili, M. H., Fisher, P. B., Subjeck, J. R., and Wang, X. Y. (2011). Targeting the immunoregulator SRA/CD204 potentiates specific dendritic cell vaccine-induced $\mathrm{T}$-cell response and antitumor immunity. Cancer Res. 71, 6611-6620.

Ying, M., and Flatmark, T. (2006). Binding of the viral immunogenic octapeptide VSV8 to native glucose-regulated protein Grp94 (gp96) and its inhibition by the physiological ligands
ATP and $\mathrm{Ca} 2+$ FEBS J. 273 , 513-522.

Conflict of Interest Statement: The authors declare that the research was conducted in the absence of any commercial or financial relationships that could be construed as a potential conflict of interest.

Received: 09 December 2011; accepted: 14 March 2012; published online: 30 March 2012.

Citation: Murshid A, Gong J and Calderwood SK (2012) The role of heat shock proteins in antigen cross presentation. Front. Immun. 3:63. doi: 10.3389/fimmu.2012.00063

This article was submitted to Frontiers in Antigen Presenting Cell Biology, a specialty of Frontiers in Immunology. Copyright (c) 2012 Murshid, Gong and Calderwood. This is an open-access article distributed under the terms of the Creative Commons Attribution Non Commercial License, which permits non-commercial use, distribution, and reproduction in other forums, provided the original authors and source are credited. 